\title{
Dirk Lewandowski, Hamburg \\ Die Macht der Suchmaschinen und ihr Einfluss auf unsere Entscheidungen
}

Wenn man die Recherche in Suchmaschinen als Vorbereitung einer Entscheidung betrachtet, kommt diesen Suchwerkzeugen aufgrund der Masse der an sie gestellten Anfragen eine nicht zu unterschätzende Bedeutung zu. Macht haben Suchmaschinen vor allem dadurch, dass sie entscheiden, was ein Nutzer zu seiner Suchanfrage zu sehen bekommt, verstärkt durch die Entscheidung, an welcher Stelle und in welcher Darstellungsform die Ergebnisse angezeigt werden. Im Suchprozess gibt es zahlreiche Stellen, an denen das Design der Suchmaschine die Entscheidung des Nutzers für oder gegen bestimmte Ergebnisse beeinflusst. Zusammen mit der externen Beeinflussung der Suchergebnisse durch sog. Suchmaschinenoptimierung ergibt sich eine Steuerung der Nutzer hin zu bestimmten Ergebnissen und Ergebnisformen. Der Artikel zeigt, wo Suchmaschinen Einfluss auf unsere Entscheidungsvorbereitung bzw. Entscheidungsfindung nehmen, an welchen Punkten dem durch einen bewussteren Umgang mit den Suchmaschinen entgegengewirkt werden kann, aber auch wo die Grenzen der eigenen Entscheidungsmöglichkeiten liegen.

Deskriptoren: Suchmaschine, Empfehlung, Ranking, Informationsvermittlung, Entscheidungshilfe

\section{The power of search engines and their influence on our} decisions

Information seeking in search engines can be regarded as a sequence of decisions a user needs to make to fulfil his information need. As search engines are the mostused service on the Internet, their importance cannot be underestimated. The power of search engines results from search engine providers' decisions on what users get to see on the results pages, including decisions on the results' positions and their display. There are many steps within the searching process where the design of the search engine can influence a user's decision for or against a certain result. Taken together with external influences of search results (e.g., through search engine optimization), users are guided towards certain results and results types, as well. This article considers the influence the design of search engines and the (assumed) intent of the search engine providers have on users' decisions within the searching process. Possible solutions for gaining more influence on the users' side are shown, but also their limitations.

Descriptors: Search engine, Raking, Suggestion, Decision support

\section{Le pouvoir des moteurs de recherche et leur influence sur nos décisions}

Si la recherche dans les moteurs de recherche est considérée dans l'optique d'une préparation pour une prise de décision, ces outils de recherche jouent un rôle à ne pas sous-estimer en raison de la masse des demandes traitées. Le pouvoir des moteurs de recherche réside dans le fait que ces derniers décident quel résultat l'utilisateur voit à l'issue de sa recherche; ce pouvoir est encore renforcé par la décision de l'endroit et de la forme de présentation des résultats. Dans le processus de recherche, la conception du moteur influence la décision de l'utilisateur pour ou contre certains résultats à de nombreux endroits. Combiné à l'influence externe des résultats de recherche par la prétendue optimisation des moteurs de recherche, ceci nous mène à un guidage des utilisateurs vers des résultats particuliers et des formes particulières de résultats. Cet article nous montre où les moteurs de recherche peuvent influencer notre préparation de prise de décision et la prise de décision elle-même, comment nous pouvons neutraliser ces effets par une utilisation plus consciente des moteurs de recherche, mais également où se trouvent les limites de nos propres choix.

Descripteurs: Moteur de recherche, Classement, Suggestion, Prise de décision

DOI 10.1515/iwp-2014-0050 


\section{Einleitung}

In diesem Aufsatz wird der Frage nachgegangen, auf welche Weise Suchmaschinen Macht ausüben und auf welchen Ebenen diese Macht die Entscheidungen der Nutzer beeinflusst. Dabei wird die Suche im Web über allgemeine Suchmaschinen betrachtet. Andere Suchanwendungen werden hier nicht berücksichtigt, auch wenn sie in speziellen Bereichen durchaus einen gewichtigen Einfluss auf die Entscheidungen der Nutzer ausüben. So ist beispielsweise an die Suche in E-Commerce-Portalen wie Ebay oder Amazon zu denken, mit der Nutzer Kaufentscheidungen vorbereiten. Während man bei den allgemeinen Suchmaschinen ein Ranking nach „objektiven Kriterien“ zumindest für wünschenswert erachtet, kommen bei denjenigen Suchanwendungen, die explizit auf einen Abverkauf von Produkten zielen, Rankingfaktoren wie die Verfügbarkeit eines Produkts oder die Marge des Händlers ins Spiel. Hier würde man also nicht per se eine Gleichbehandlung aller Objekte im Index annehmen.

Im Gegensatz dazu ist es ein wesentlicher Faktor der Websuchmaschinen, dass sie nur als Vermittler von Informationen auftreten, nicht jedoch als Anbieter von Inhalten. Wie zu zeigen sein wird, können Suchmaschinen heute jedoch nicht als „neutrale Informationsvermittler“ angesehen werden (wenn das denn jemals möglich war), sondern als Informationsvermittler mit eigenen (kommerziellen) Interessen. Es ist daher $\mathrm{zu}$ fragen, inwieweit Suchmaschinen die Entscheidungen der Nutzer in diesem Sinne beeinflussen (können).

Suchmaschinen sind heute die dominierenden Werkzeuge zur Informationssuche im Web. Andere Suchansätze (etwa beschrieben in Griesbaum, Bekavac, \& Rittberger, 2009) spielen kaum mehr eine Rolle und die Websuche ist neben E-Mail weiterhin die beliebteste Anwendung des Internet (van Eimeren \& Frees, 2013). Auch die schiere Masse der an die allgemeinen Suchmaschinen gestellten Anfragen verdeutlicht ihre Bedeutung als dominierendes Werkzeug der Websuche. Die aktuellsten Zahlen für Deutschland stammen aus dem Jahr 2009 und zeigen, dass schon damals in einem einzigen Monat 5,6 Milliarden Suchanfragen an die allgemeinen Suchmaschinen gestellt wurden (ComScore, 2010). Dies sind umgerechnet pro Sekunde mehr als 2.000 Suchanfragen.

In diesem Aufsatz wird die Websuche als Prozess verstanden, der bei der Entscheidungsfindung unterstützt bzw. eine Entscheidung vorzubereiten hilft. Andere Zwecke (wie beispielsweise die Suche zur Unterhaltung, vgl. Elsweiler, Wilson, \& Lunn, 2011; (Waller, 2011) werden hier nicht betrachtet. Ausgeklammert werden auch Suchanfragen, die nicht der Vorbereitung einer nennens- werten Entscheidung dienen. Damit werden beispielsweise navigationsorientierte Suchanfragen (Broder, 2002) und Suchanfragen, die sich auf sog. Trivia beziehen (Lewandowski, 2014), ausgeschlossen.

\section{Der Suchmaschinenmarkt und die Macht der Suchmaschinen}

Betrachtet man den Suchmaschinenmarkt, so ist man erst einmal mit einer Vielzahl von Anbietern konfrontiert. Allerdings unterhalten nur wenige Suchmaschinen einen eigenen Index, d. h. erfassen selbst die Daten des Webs und bereiten diese auf. Die meisten Anbieter haben dagegen Partnerschaften mit einem der Anbieter mit eigenem Index (v.a. Google) geschlossen und zeigen dessen Ergebnisse in einem eigenen Interface an (vgl. Lewandowski, 2013). Dieses Modell des sog. Partner-Index hat zu einer Reduzierung der Anzahl wirklich unterschiedlicher Suchmaschinen geführt; das prominenteste Beispiel ist sicherlich die Kooperation zwischen Yahoo und Microsoft, in deren Rahmen Yahoo seine eigene Suchmaschine aufgegeben hat und seitdem Ergebnisse von Microsofts Suchmaschine Bing anzeigt.

So findet sich heute auf dem Suchmaschinenmarkt nur vordergründig eine Vielzahl von Anbietern; sieht man hinter die Kulissen, reduziert sich die Anbieterzahl erheblich. Hinzu kommt, dass viele kleine Anbieter keine konkurrenzfähigen Suchmaschinen anbieten und sich daher als echte Alternativen nicht eignen. Doch auch wenn echte Alternativen in höherer Zahl vorhanden wären, so zeigtt doch die Suchmaschinennutzung ein eindeutiges Bild: Google dominiert den Markt, andere Suchmaschinen werden kaum genutzt. Die Macht von Suchmaschinen ergibt sich nun aus ihrer Rolle als Filter, der die Masse an (potentiell relevanten) Inhalten in eine Reihenfolge bringt. Man mag hier von „Gatekeepern“ sprechen (Machill \& Beiler, 2002), auch wenn die einfache Übertragbarkeit dieses Begriffs von den Massenmedien, die ein bestimmtes Programm senden, auf Suchmaschinen, bei denen der Nutzer mit jeder Suchanfrage ein neues „Programm“ bestimmt, fraglich ist (Röhle, 2010). Vielfach wird der Wunsch nach „neutralen Suchmaschinen“ geäußert. Eine solche Suchmaschine gibt es allerdings nicht und kann es nicht geben, weil jede Suchmaschine in bestimmter Weise Verzerrungen ausgesetzt ist. Weber (2011, S. 278) benennt drei Bereiche, in denen sich diese Verzerrungen (bias) ergeben:

1. Implementierung (in einem weiten Sinne) der jeweiligen Suchmaschine

2. Verhalten der Anbieter von Inhalten im Internet

3. wie Suchmaschinen genutzt werden 


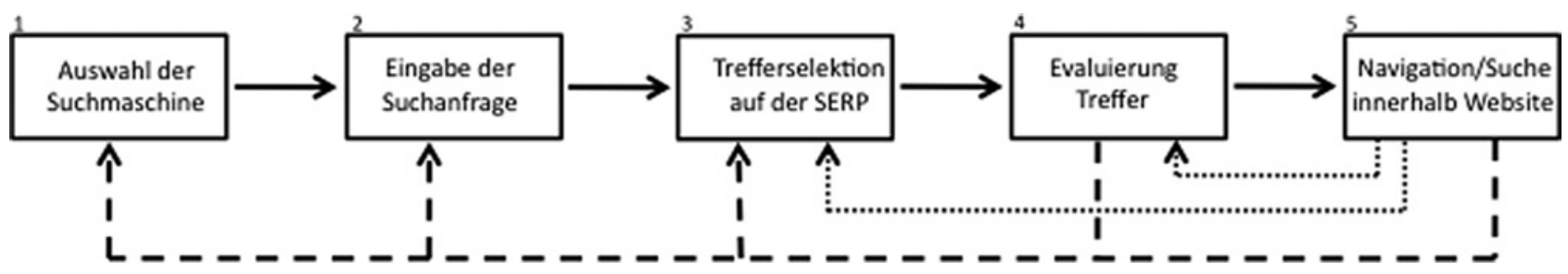

Abbildung 1: Prozess der Websuche (aus Lewandowski, 2012, S. 103).

Im Folgenden soll es vor allem um den Bereich Implementierung gehen. Dabei wird die Ansicht vertreten, dass die - ohnehin nicht vermeidbaren - Verzerrungen erst durch die Dominanz eines einzelnen Anbieters zu einem erheblichen Problem geworden sind. Hätten wir eine Vielfalt von Anbietern auf dem Suchmaschinenmarkt, die alle auf unterschiedliche Weise verzerrte Suchergebnisse ausgeben würden, könnte mittels verzerrter Ergebnisse nur ein geringer Einfluss ausgeübt werden und Verzerrungen wären im direkten Vergleich leichter zu entdecken.

Verzerrungen werden zum Problem durch die Verbindung von drei Faktoren:

1. Der Dominanz des Modells ,algorithmische Web-Suchmaschine“ gegenüber anderen Methoden zum Auffinden von Informationen im World Wide Web

2. der Dominanz von Google in diesem Bereich

3. dem Verhalten der Suchmaschinennutzer (kurze Suchanfragen, kaum systematische Ergebnissichtung, geringe Kenntnisse über Suchmaschinen)

Die Macht der Suchmaschinen ist dabei auf zwei Ebenen erkennbar. Zum einen haben Suchmaschinen Macht über das, was überhaupt in den Index (also den der Suchmaschine zugrunde liegenden Datenbestand) aufgenommen wird. Dabei gibt es technische Probleme, die die Erstellung eines vollständigen und aktuellen Index verhindern (s. u. a. Broder et al., 2000; Lewandowski, 2008a; Bergman, 2001; Lewandowski \& Mayr, 2006). Außerdem erfolgt ein demokratisch legitimierter Ausschluss von Inhalten (beispielweise in Deutschland verbotene Seiten, die den Holocaust leugnen), aber auch ein willkürlicher Ausschluss von Inhalten (d. h. diese werden vom jeweiligen Suchmaschinenbetreiber nach nicht öffentlich gemachten Kriterien ausgeschlossen). ${ }^{1}$

1 Man muss hier bedenken, dass eine Suchmaschine, die keine Inhalte in der Indexierung ausschließen würde, weder wünschenswert noch praktikabel wäre. Dies liegt vor allem in der Masse der SpamInhalte begründet, also Dokumenten, die ohne inhaltlichen Mehrwert allein zur Werbung und/oder zur Irreführung der Nutzer erstellt werden. Die Suchmaschinenbetreiber machen allerdings nicht öffentlich, wo ihre Grenzen zwischen akzeptablen Dokumenten und unerwünschten Inhalten liegen.
Die zweite Ebene der Macht der Suchmaschinen liegt in der Macht darüber, was Nutzer zu sehen bekommen. Diese Macht wird ausgeübt durch die Reihung der Dokumente (Ranking), durch die Anordnung der Suchergebnisseiten (vgl. Höchstötter \& Lewandowski, 2009) und durch das Einblenden von direkten Antworten bzw. Fakteninformationen auf den Suchergebnisseiten.

\section{Entscheidungen im Suchprozess}

Abbildung 1 zeigt den typischen Suchprozess einer Websuche. Zuerst sollen die Schritte dieses Prozesses kurz beschrieben werden, bevor im Detail die möglichen Einflussnahmen der Suchmaschinen(betreiber) auf den unterschiedlichen Stufen diskutiert werden.

Im ersten Schritt wird eine passende Suchmaschine ausgewählt. Dort wird dann im zweiten Schritt eine Suchanfrage eingegeben bzw. während der Eingabe einer Suchanfrage einer der angebotenen Suchvorschläge (vgl. Lewandowski \& Quirmbach, 2013) ausgewählt, was zum Absenden der Suchanfrage führt. Im dritten Schritt wählt der Nutzer aus den angebotenen Treffer einen aus; im vierten Schritt wird das Trefferdokument auf seine Eignung geprüft. Anschließend erfolgt eventuell innerhalb der angewählten Website eine weitere Navigation bzw. eine Suche innerhalb dieser Website.

Der Suchprozess ist nicht nur als lineare Abfolge von Schritten zu sehen, sondern zwischen den Schritten kann gesprungen werden (in der Abbildung durch die unterbrochenen Pfeile dargestellt). Dabei kann ein Nutzer beispielweise von der Evaluierung eines Trefferdokuments zur Suchergebnisseite der Suchmaschine zurückkehren, aber auch direkt zur Sucheingabe, um seine Suchanfrage zu verfeinern oder durch eine andere Suchanfrage zu ersetzen.

\section{Auswahl der Suchmaschine}

Die Auswahl einer Suchmaschine geschieht vordergründig erst einmal über das Ansteuern einer bestimmten 
URL (also beispielsweise www.google.de). Hiermit würde ein Nutzer bei jedem neuen Suchvorgang eine aktive Entscheidung für eine Suchmaschine treffen, auch wenn diese Entscheidung von Gewohnheiten und Kenntnissen beeinflusst sein mag.

Allerdings gibt es Faktoren, die einen erheblichen Einfluss auf die Nutzung bestimmter Suchmaschinen haben. Dazu zählen die Voreinstellung einer Suchmaschine im Suchfeld des Browsers, die Voreinstellung einer Suchmaschine als Startseite im Browser sowie die Voreinstellung einer Suchmaschine in der URL-Leiste. ${ }^{2}$ Hier kann man dann nicht mehr von einer aktiven Auswahl einer Suchmaschine zum Beginn eines jeden Suchvorgangs sprechen, sondern die Entscheidung für eine andere als die voreingestellte Suchmaschine ist als Entscheidung gegen die Standardsuchmaschine zu betrachten.

Es ist daher nicht verwunderlich, dass Suchmaschinenbetreiber Verträge mit Browserherstellern abschließen, nach denen dann eine bestimmte Suchmaschine voreingestellt wird. Für einige Browser ist dies auch das wesentliche Geschäftsmodell: So bezieht die Mutter des Firefox-Browsers, die Mozilla Foundation, den bei weitem wesentlichen Teil (etwa 90 Prozent) ihrer Einnahmen aus einer Kooperation mit Google (Protalisnki, 2013). Google bezahlt für jede Firefox-Installation, bei der Google als Startseite, in der URL-Leiste und/oder im Suchfeld voreingestellt ist. Ähnliche Kooperationen gibt es zwischen Suchmaschinen und Herstellern von Mobiltelefonen. Ebenso integriert Microsoft seine Suchmaschine Bing in Windows; Google integriert seine Suchmaschine in den eigenen Browser Chrome.

\section{Eingabe der Suchanfrage}

Die nächsten Entscheidungen sind bei der Eingabe der Suchanfrage zu treffen. Suchanfragen lassen sich auf unterschiedliche Weisen stellen, zum Beispiel als Frage, als Aneinanderreihung von Stichwörtern oder als mittels Operatoren und Kommandos qualifiziertes Suchargument.

Während alle diese Formen gebräuchlich sind und von den Suchmaschinen unterstützt werden, werden Suchanfragen in den bei weitem häufigsten Fällen schlicht als Aneinanderreihung von Wörtern gestellt. Dabei gilt nach wie vor die von Machill et al. (2003, S. 169) getroffene Aussage: „Die meisten Nutzer sind nicht willens, bei der Formulierung ihres Suchziels allzu viel kognitive und zeitliche Energie aufzuwenden." Suchanfra-

2 Bei einigen Browsern, beispielsweise Safari, sind Suchfeld und URL-Leiste zusammengefasst. gen sind nach wie vor kurz (oft bestehen sie nur aus einem oder wenigen Wörtern; s. Höchstötter \& Koch, 2009), Nutzer erwarten anstelle einer komplexen Anfrageformulierung eine hohe Interpretationsleistung der Suchmaschine, die antizipieren soll, was mit einer aktuellen Anfrage gemeint ist (Lewandowski, 2011). Da in vielen Fällen das Informationsbedürfnis nur unzureichend zum Ausdruck gebracht wird, entsteht häufig ein „Übersetzungsproblem“ von einem Informationsbedürfnis des Nutzers in seine Suchanfrage.

Natürlich kann schon die Interpretation der Suchanfrage (und im folgenden Schritt dann die Anzeige von $z u$ dieser Anfrageinterpretation passenden Treffern) weitere Entscheidungen des Nutzers beeinflussen. Doch auch schon im Schritt der Anfrageformulierung geben Suchmaschinen Hilfestellungen in Form von Suchvorschlägen während der Eingabe („Autocomplete“, s. Lewandowski \& Quirmbach, 2013). Auf der einen Seite wird damit eine Unterstützung bei der Formulierung der Suchanfrage gegeben (beispielsweise durch Rechtschreibkorrekturen oder die Ergänzung weiterer, qualifizierender Suchwörter), auf der anderen Seite lassen sich Nutzer mittels der Suchvorschläge aber auch lenken bzw. es ist Suchmaschinen möglich, die Entscheidungen in der Formulierung von Suchanfragen bei ihren Nutzern zu beeinflussen.

\section{Trefferselektion auf der Suchergebnisseite}

Auf der Suchergebnisseite (,Search Engine Results Page“, SERP) werden die Elemente präsentiert, aus denen ein Nutzer die nach der Eingabe einer Suchanfrage ermittelten Treffer auswählen kann. Unter Elementen sind hier sowohl Suchergebnisse, Werbetreffer, Fakteninformationen und Navigationselemente zu verstehen. Dies bedeutet, dass ein Nutzer 1. auf einer Suchergebnisseite nicht unbedingt einen Treffer anklicken muss (sondern beispielsweise den Link auf die nächste Suchergebnisseite) und 2. nicht unbedingt überhaupt etwas auf der Suchergebnisseite angeklickt wird (beispielsweise, wenn die gewünschte Fakteninformation schon auf der Suchergebnisseite selbst präsentiert wird).

Suchergebnisseiten teilen sich in den sog. sichtbaren und den sog. unsichtbaren Bereich (Höchstötter \& Lewandowski, 2009, S. 1798). Bei dem sichtbaren Bereich handelt es sich um den Bereich, der direkt auf dem Bildschirm zu sehen ist, ohne dass gescrollt werden muss. Je nach Endgerät und Bildschirmgröße kann die Größe dieses Bereichs sehr unterschiedlich ausfallen. Zahlreiche Studien haben gezeigt, dass sich Nutzer vornehmlich für 
Elemente entscheiden, die im sichtbaren Bereich der Suchergebnisseite liegen (Cutrell \& Guan, 2007; Granka, Hembrooke, \& Gay, 2006). Der unsichtbare Bereich (,scroll area“) findet dagegen weit weniger Beachtung.

Die Suchergebnisseite enthält die sog. organischen Ergebnisse, wobei es sich um Ergebnisse handelt, die aus dem allgemeinen Web-Index der Suchmaschine generiert werden. Diese Suchergebnisse sind zunächst einmal „neutral“, d.h. jedes Dokument hat potentiell die gleiche Chance, in den Suchergebnissen aufzutauchen, da alle Dokumente nach demselben Verfahren gerankt werden. Eine Beeinflussung findet allein von außen, in diesem Fall durch Suchmaschinenoptimierung, statt. Dabei ist unklar, inwieweit von Nutzern erkannt wird bzw. erkannt werden kann -, bei welchen Ergebnissen es sich um optimierte Ergebnisse handelt, zumal die Abgrenzung zwischen einer „automatischen“ Optimierung während der Texterstellung (beispielsweise durch die Wahl aussagekräftiger Titel und Überschriften) und professionellen Optimierungsmaßnahmen fließend ist.

Sieht man von der Suchmaschinenoptimierung ab, werden Nutzer vor allem durch die Reihung der Ergebnisse beeinflusst, was in zahlreichen Studien nachgewiesen wurde (u. a. Keane, O’Brien, \& Smyth, 2008).

Neben den organischen Suchergebnissen werden auf den Suchergebnisseiten in vielen Fällen auch Anzeigen dargestellt. Eine Besonderheit ist dabei, dass die Anzeigen im Kontext der jeweiligen Suchanfrage stehen und als Text, der in seiner Darstellung den Trefferbeschreibungen der organischen Treffern ähnelt, dargestellt werden. Man kann sich die Auslieferung der Anzeigen analog zur Auslieferung der organischen Suchergebnisse vorstellen, wobei der Datenbestand jeweils ein komplett verschiedener ist: Hier eine Datenbank, die von den Anzeigenkunden mit URLs und kurzen Beschreibungen ,gefüttert" wird, dort ein automatisch generierter Bestand von Dokumenten, die über das World Wide Web verstreut sind. Auf der Suchergebnisseite nun werden die Textanzeigen und die organischen Treffer miteinander kombiniert; sie erhalten jedoch jeweils abgegrenzte Bereiche innerhalb der Suchergebnisseite. Untersuchungen deuten darauf hin, dass nicht alle Nutzer - und nicht alle Nutzer in allen Fällen - zwischen den organischen Ergebnissen und den Textanzeigen unterscheiden können (Bundesverband Digitale Wirtschaft, 2009). Dieses mangelnde Wissen führt dazu, dass Nutzer nicht in allen Fällen eine Entscheidung treffen, die auf einer korrekten Einschätzung der Herkunft der Treffer beruht.

Ein Sonderfall der organischen Treffer sind die sog. Universal-Search-Ergebnisse. Bei Universal Search handelt es sich um ein Konzept, bei dem Suchergebnisse aus unterschiedlichen Kollektionen (Datenbeständen) abgefragt und auf einer Suchergebnisseite zusammengeführt werden. Dabei dient die Liste der organischen Treffer als Basis; die Universal-Search-Ergebnisse (bspw. Nachrichten, Videos) werden innerhalb der Liste in besonderer Form (bspw. mit Vorschaubildern) dargestellt. Sie durchbrechen damit die typische Listendarstellung auf den Suchergebnisseiten und lenken durch ihre besondere Darstellung die Blicke (und in deren Folge auch die Klicks) der Nutzer. Dies mag unproblematisch sein, solange die Ergebnisse aus den einzelnen Kollektionen so erzeugt werden wie organische Ergebnisse, d. h. jedes Dokument die gleiche Chance hat, als Ergebnis einer Suchanfrage angezeigt zu werden. Allerdings ist dies nicht der Fall, und gerade das Kartellverfahren der Europäischen Union gegen Google hat gezeigt, dass Google in den UniversalSearch-Ergebnissen seine eigenen Angebote bevorzugt.

Eine neuere Entwicklung ist, dass zunehmend Fakteninformationen auf den Suchergebnisseiten dargestellt werden. Zwar gab es bereits in der Vergangenheit Fakteninformationen zu einfachen Fragen oberhalb der Trefferlisten zu sehen, inzwischen hat sich allerdings mit dem sog. „Knowledge Graph“ (so die Bezeichnung bei Google) eine Zusammenstellung von Fakteninformationen auf den Suchergebnisseiten durchgesetzt. Wird also nur ein Faktum gesucht und dieses taucht innerhalb des Knowledge Graph auf, ist kein Klick mehr auf ein Ergebnis nötig. Zwar gibt es noch keine veröffentlichten Studien zu diesem Thema, allerdings ist anzunehmen, dass viele Nutzer, sofern ihre Frage mittels des Knowledge Graph beantwortet wird, keine Dokumente mehr sichten. Was dabei verloren geht, sind Kontextinformationen (wer nennt in welchem Zusammenhang ein Faktum); Nutzer geben damit die Entscheidung über die Richtigkeit bzw. Vertrauenswürdigkeit ein Stück weit weiter an die Suchmaschine ab.

\section{Evaluierung der Treffer}

Wurde ein Treffer auf der Suchergebnisseite ausgewählt, gelangt der Nutzer zum Trefferdokument selbst. Dort wird die Entscheidung getroffen, ob das Dokument wert ist, genauer gesichtet zu werden oder nicht. Erscheint das Dokument nicht brauchbar, erfolgt meist eine Rückkehr auf die Suchergebnisseite. Die Trefferbeschreibungen auf den Suchergebnisseiten geben zwar einen Anhaltspunkt für die Relevanz der dahinterliegenden Treffer; in einem gewissen Anteil der Fälle führen allerdings relevante Beschreibungen zu irrelevanten Treffern bzw. als irrelevant erachtete Beschreibungen führen zu relevanten Treffern (Lewandowski, 2008b). 


\section{Navigation/Suche innerhalb der Website}

Nachdem auf einem Trefferdokument die Entscheidung getroffen wurde, nicht auf die Suchergebnisseite zurückzukehren oder die Suche abzubrechen, kann entweder das Dokument genauer angesehen werden und/oder der Nutzer navigiert innerhalb der Website bzw. führt über ein Suchfeld dort eine weitere, site-interne Suche aus. Innerhalb dieser Suche sind dann ggf. ähnliche Entscheidungen wie in der beschriebenen Suche mit einer Suchmaschine $\mathrm{zu}$ treffen.

\section{Die Entscheidungsfreiheit der Nutzer}

Natürlich haben wir mit Suchmaschinen Zugriff auf eine schier unüberschaubare Menge von Informationen, und das Verdienst der Suchmaschinen bei der Erschließung des Webs ist nicht hoch genug einzuschätzen. Dies schließt aber nicht aus, Suchmaschinen(betreiber) auch zu kritisieren bzw. es ist nicht legitim, jede Kritik an Praktiken der Suchmaschinenbetreiber mit einem Verweis auf diese unbestrittenen Verdienste abzutun.

Ein häufig vorgebrachtes Argument ist, dass Suchmaschinennutzer in ihren Entscheidungen frei seien und aus einer riesigen Anzahl von Ergebnissen, die eine Suchmaschine zu so gut wie jeder Suchanfrage anzeigen würde, auswählen könnten. Das Ranking sei nur eine $A n$ ordnung der Treffer; die Treffermenge bestimme der Nutzer durch seine Suchanfrage selbst.

Dies trifft allerdings nicht zu. Zum einen ist es technisch gar nicht möglich, sich sämtliche Suchergebnisse anzusehen. Keine der gängigen Suchmaschinen zeigt zu einer Anfrage mehr als 1.000 Treffer an. Man kann zwar auf den Trefferseiten immer weiterblättern, weiter als zum tausendsten Treffer kommt man aber nie. Insofern sind auch die oberhalb der Trefferlisten angegebenen Ergebniszahlen („Treffer 1-10 von...“) irreführend, da sie suggerieren, man könne theoretisch all diese Treffer auch ansehen.

Zum zweiten wäre es selbstverständlich nicht praktikabel, sich alle Treffer einer umfangreichen Treffermenge anzusehen. Wir müssen uns auf das Ranking verlassen; die Treffermenge, aus der wir einen Treffer auswählen, wird immer beschränkt sein - und zwar nicht dadurch, dass es nicht mehr Treffer gibt, sondern dadurch, dass wir nur begrenzte Aufnahmekapazitäten haben.

Und drittens wäre das Sichten aller Treffer nicht effizient. Als Nutzer können wir gar nicht anders, als uns (zumindest ein Stück weit) auf das von den Suchmaschinen vorgegebene Ranking zu verlassen. Dabei gehen wir davon aus, dass unsere Lieblingssuchmaschine das „Versprechen des Rankings“ (die für uns im aktuellen Kontext besten Dokumente oben zu listen) auch tatsächlich erfüllen kann.

Große Treffermengen haben auf der einen Seite mit der Größe des World Wide Web zu tun. Wenn wir riesige Datenbestände durchsuchen, müssen wir auch große Treffermengen erwarten. Zum anderen hat die Größe der Treffermengen aber auch mit der Qualität der Suchanfragen zu tun. Gerade die hauptsächlich kurzen und kaum qualifizierten Anfragen an Suchmaschinen führen zu großen Treffermengen, bei denen wir uns umso mehr auf das Ranking der Suchmaschine verlassen müssen. Insofern tritt neben die Steuerung der Entscheidung für oder gegen einen Treffer durch Suchmaschinenbetreiber, Inhalteanbieter und Suchmaschinenoptimierer eine selbstverschuldete Unmündigkeit, verursacht durch mangelnde Informationskompetenz.

\section{Der Wandel der Trefferpräsentation und sein Einfluss auf die Entscheidungen der Nutzer}

Oben wurde der aktuelle Stand der Trefferpräsentation auf den Suchergebnisseiten beschrieben. Man kann daran ablesen, dass sich die Ergebnispräsentation von der einfachen gereihten Liste hin zu einer komplexen Zusammenstellung von Ergebnissen unterschiedlicher Art gewandelt hat.

Dieser Wandel lässt sich in mehreren Stufen beschreiben:

1. Ergebnislisten: In der einfach gereihten Ergebnisliste bestehen Positionseffekte, die schlicht durch die Anordnung der Ergebnisse und die Lesereihenfolge verursacht werden. Die Darstellung der einzelnen Ergebnisse ist weitgehend gleich.

2. Universal-Search-Ergebnisseiten: Die Listendarstellung wird grundsätzlich beibehalten, zusätzlich wird die Aufmerksamkeit der Nutzer durch grafische Elemente und Hervorhebungen gelenkt.

3. Universal-Search-Ergebnisseite mit Knowledge Graph und erweiterten Trefferbeschreibungen: Die Listendarstellung wird immer weiter aufgebrochen; auch organische Treffer werden unterschiedlich dargestellt; die Ergebnisseiten bestehen nicht mehr notwendigerweise aus Verweisen auf Dokumente.

Betrachtet man nun diesen Wandel mit dem Blick auf die Entscheidung des Nutzers für einen Treffer, so zeigt sich 
eine zunehmende Lenkung der Aufmerksamkeit - für den Nutzer wird die Suchergebnisseite gleichzeitig attraktiver (größere Vielfalt, ansprechende Gestaltung) und undurchschaubarer.

\section{Der Einfluss der Suchmaschinenoptimierung}

Unter Suchmaschinenoptimierung („Search Engine Optimization“, SEO) versteht man alle Maßnahmen, um die Sichtbarkeit von Dokumenten für Suchmaschinen zu erhöhen. Es gibt inzwischen kaum noch kommerzielle Unternehmen, die im Internet aktiv sind und keine SEOMaßnahmen ergreifen; der Einfluss auf die Suchergebnisse im kommerziellen Bereich ist evident, und Suchmaschinenoptimierung hat sich mittlerweile als Dienstleistung etabliert.

Ein Trend, der in den letzten Jahre schon beobachtet werden konnte, sich aber noch weiter verstärken dürfte, ist der Einsatz von Maßnahmen der Suchmaschinenoptimierung auch im nicht direkt kommerziellen Bereich. So ist SEO für journalistische Inhalte mittlerweile eine Selbstverständlichkeit, es gibt aber auch Suchmaschinenoptimierung für Nichtregierungsorganisationen, Suchmaschinenoptimierung als PR-Maßnahme und Suchmaschinenoptimierung für wissenschaftliche Aufsätze („Academic Search Engine Optimization“, s. Beel, Gipp, \& Wilde, 2010). All diesen Bereichen ist gemein, dass hier nicht optimiert wird, um ein Produkt oder eine Dienstleistung zu verkaufen, sondern dass direkt das Produkt also der Content - dahingehend optimiert wird, dass er besser auffindbar wird. Die Suchergebnisseiten werden damit zunehmend zum Schlachtfeld für Public Relations.

\section{Fazit}

In diesem Artikel wurde dargestellt, dass Nutzer an verschiedenen Stellen im Suchprozess Entscheidungen treffen, und dass diese Entscheidungen zumindest zu einem Teil von der Art und Weise, wie der Suchprozess seitens der Suchmaschinenbetreiber gestaltet wird, beeinflusst werden. Dabei ergänzen sich die Designentscheidungen der Suchmaschinenbetreiber und die selbstverschuldete Informationsinkompetenz der Nutzer gegenseitig. Es besteht eine erhebliche Diskrepanz zwischen der massenhaften Nutzung von Suchmaschinen sowie der Bedeutung der Suchmaschinenrecherche auf der einen Seite und den Kenntnissen der Nutzer auf der anderen Seite. Diese mangelhaften Kenntnisse beziehen sich sowohl auf die von den Suchmaschinen gebotenen Suchmöglichkeiten, welche bei weitem nicht ausgenutzt werden, als auch auf die Unkenntnis der Funktionsweise der Suchmaschinen und ihrer Geschäftsmodelle.

Es ist zu hoffen, dass die Bedeutung des Themas Informationskompetenz in Hinblick auf Suchmaschinen erkannt wird und Nutzer ein Verständnis der Informationswerkzeuge, die sie schließlich meist täglich benutzen, erlangen. Dies bedingt allerdings auch eine bessere Durchdringung des Themas Suchmaschinen bzw. Suche im Internet durch verstärkte Anstrengungen in der Forschung.

Und schließlich ist zu hoffen, dass eine der großen Fehlannahmen über Suchmaschinen endlich ausgeräumt wird: Suchmaschinen sind eben keine neutralen Vermittler von Informationen, sondern ihre Betreiber verfolgen Interessen, die sie auch über den Suchprozess und die Suchergebnisseiten ausspielen.

\section{Literatur}

Beel, J., Gipp, B. \& Wilde, E. (2010). Academic Search Engine Optimization (ASEO): Optimizing Scholarly Literature for Google Scholar \& Co. Journal of Scholarly Publishing, 41(2), 176-190.

Bergman, M. K. (2001). The deep Web: Surfacing hidden value. Journal of Electronic Publishing, 7(1), 1-17.

Broder, A. (2002). A taxonomy of web search. ACM Sigir Forum, 36(2), 3-10.

Broder, A., Kumar, R., Maghoul, F., Raghavan, P., Rajagopalan, S., Stata, R., ... Wiener, J. (2000). Graph structure in the web. Computer Networks, 33(1-6), 309-320.

Bundesverband Digitale Wirtschaft. (2009). Nutzerverhalten auf Google-Suchergebnisseiten: Eine Eyetracking-Studie im Auftrag des Arbeitskreises Suchmaschinen-Marketing des Bundesverbandes Digitale Wirtschaft (BVDW) e. V. http://bvdw.org/ fileadmin/bvdw-shop/ets08.pdf [ 28.6.2014].

ComScore. (2010). comScore Reports Global Search Market Growth of 46 Percent in 2009. http://comscore.com/Press_Events/ Press_Releases/2010/1/Global_Search_Market_Grows_46_Percent_in_2009 [ 28.6.2014].

Cutrell, E. \& Guan, Z. (2007). What are you looking for? An eye-tracking study of information usage in Web search. In Proceedings of the SIGCHI Conference on Human Factors in Computing Systems (CHI 2007) (S. 407-416). New York: ACM.

Elsweiler, D., Wilson, M. L. \& Lunn, B. K. (2011). Understanding Casual-Leisure Information Behaviour. In A. Spink \& J. Heinström (Hrsg.), New Directions in Information Behaviour (S. 211-241). Bingley: Emerald Group Publishing.

Granka, L., Hembrooke, H. \& Gay, G. (2006). Location location location: Viewing patterns on WWW pages. In Proceedings of the 2006 Symposium on Eye Tracking Research \& Applications (Vol. 2005, S. 43-43). New York: ACM.

Griesbaum, J., Bekavac, B. \& Rittberger, M. (2009). Typologie der Suchmaschine im Internet. In D. Lewandowski (Hrsg.), Handbuch Internet-Suchmaschinen (S. 18-52). Heidelberg: Akademische Verlagsgesellschaft Aka. 
Höchstötter, N. \& Koch, M. (2009). Standard parameters for searching behaviour in search engines and their empirical evaluation. Journal of Information Science, 35(1), 45-65.

Höchstötter, N. \& Lewandowski, D. (2009). What users see Structures in search engine results pages. Information Sciences, 179(12), 1796-1812.

Keane, M. T., O’Brien, M. \& Smyth, B. (2008). Are people biased in their use of search engines? Communications of the ACM, 51(2), 49-52.

Lewandowski, D. (2008a). A three-year study on the freshness of Web search engine databases. Journal of Information Science, 34, 817-831.

Lewandowski, D. (2008b). The retrieval effectiveness of web search engines: considering results descriptions. Journal of Documentation, 64(6), 915-937.

Lewandowski, D. (2011). Query Understanding. In D. Lewandowski (Hrsg.), Handbuch Internet-Suchmaschinen 2: Neue Entwicklungen in der Web-Suche (S. 55-75). Heidelberg: Akademische Verlagsgesellschaft AKA.

Lewandowski, D. (2012). Informationskompetenz und das Potenzial der Internetsuchmaschinen. In W. Sühl-Strohmenger (Hrsg.), Handbuch Informationskompetenz (S. 101-109). Berlin: De Gruyter.

Lewandowski, D. (2013). Suchmaschinenindices. In D. Lewandowski (Hrsg.), Handbuch Internet-Suchmaschinen 3: Suchmaschinen zwischen Technik und Gesellschaft (S. 143-161). Berlin: Akademische Verlagsgesellschaft AKA.

Lewandowski, D. (2014). Wie lässt sich die Zufriedenheit der Suchmaschinennutzer mit ihren Suchergebnissen erklären? In H. Krah \& R. Müller-Terpitz (Hrsg.), Suchmaschinen (S. 35-52). Münster: LIT.

Lewandowski, D. \& Mayr, P. (2006). Exploring the academic invisible web. Library Hi Tech, 24(4), 529-539.

Lewandowski, D. \& Quirmbach, S. (2013). Suchvorschläge während der Eingabe. In D. Lewandowski (Hrsg.), Handbuch InternetSuchmaschinen 3: Suchmaschinen zwischen Technik und Gesellschaft (S. 273-298). Berlin: Akademische Verlagsgesellschaft AKA.

Machill, M. \& Beiler, M. (2002). Suchmaschinen als Vertrauensgüter. Internet-Gatekeeper für die Informationsgesellschaft? In D. Klumpp, H. Kubicek, A. Roßnagel, \& W. Schulz (Hrsg.), Informationelles Vertrauen für die Informationsgesellschaft (S. 159-172). Heidelberg: Springer.
Protalisnki, E. (2013). Mozilla's reliance on Google is increasing: $90 \%$ of 2012 revenue came from that one source. The Next Web. Retrieved from http://thenextweb.com/insider/2013/11/21/ mozillas-reliance-google-increasing-90-2012-revenue-came-onesource/ [28.6.2014].

Röhle, T. (2010). Der Google-Komplex: Über Macht im Zeitalter des Internets. Bielefeld: Transcript.

Van Eimeren, B. \& Frees, B. (2013). Rasanter Anstieg des Internetkonsums - Onliner fast drei Stunden täglich im Netz. Media Perspektiven, (7-8), 358-372.

Waller, V. (2011). Not Just Information : Who Searches for What on the Search Engine Google? Journal of the American Society for Information Science and Technology, 62(4), 761-775.

Weber, K. (2011). Search Engine Bias. In D. Lewandowski (Hrsg.), Handbuch Internet-Suchmaschinen 2: Neue Entwicklungen in der Web-Suche (S. 265-285). Heidelberg: Akademische Verlagsanstalt AKA.

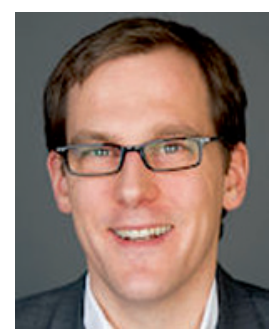

Prof. Dr. Dirk Lewandowski

Hochschule für Angewandte Wissenschaften Hamburg

Fakultät Design, Medien und Information

Finkenau 35

22081 Hamburg

dirk.lewandowski@haw-hamburg.de

www.bui.haw-hamburg.de/lewandowski.html

Dirk Lewandowski ist Professor für Information Research \& Information Retrieval an der Hochschule für Angewandte Wissenschaften Hamburg. Vorher war er unabhängiger Berater im Themenbereich Suchmaschinen und Information Retrieval sowie Lehrbeauftragter an der Universität Düsseldorf. Dr. Lewandowskis Forschungsinteressen sind Web Information Retrieval, Qualitätsfaktoren von Suchmaschinen, das Rechercheverhalten der Suchmaschinen-Nutzer sowie die gesellschaftlichen Auswirkungen des Umgangs mit den Web-Suchmaschinen. Zu seinen Veröffentlichen gehören neben den Büchern „Web Information Retrieval“ und „Handbuch Internet-Suchmaschinen" zahlreiche Aufsätze in deutschen und internationalen Fachpublikationen. 